\title{
Microprocessor Controlled Tonometry
}

\author{
J. DRAEGER, E. RUMBERGER, J. DAUPER, C. DEUTSCH
}

Hamburg, W. Germany

\begin{abstract}
Summary
Microprocessors have enabled new techniques to be applied to the design of tonometers. Three new microprocessor controlled tonometers, one operating to the principle of Mackay-Marg (Tono-Pen) and two non-contact airstream tonometers (Pulsair and CT-10) have been examined using as a reference Goldmann and the Hand Held Applanation Tonometers (Draeger-HAT) which used the Goldmann applanation principle. The non-contact tonometers produce a conically formed airstream with top speeds of $90 \mathrm{~m} / \mathrm{s}(\mathrm{CT}-10)$ and $70 \mathrm{~m} / \mathrm{s}$ (Pulsair) which at the moment of pressure measurement applanates the cornea exactly with a diameter of $4 \mathrm{~mm}$. The readings of the microprocessor controlled tonometers correlate well with the readings of HAT (Tono-Pen $r=0.8414$ and $s=3.783, C T-10 r=0.8606$ and $s=4.5477$, Pulsair $r=0.8968$ and $s=3.2641$, where $r$ is the correlation coefficient and $s$ is the standard deviation in $\mathrm{mmHg}$ ). Repeated measurements with one tonometer give the following standard deviations (HAT 1.6617, Tono-Pen 3.4969, CT-10 2.9179, Pulsair 2.9434). Although the three new instruments are able to measure the intraocular pressure in a wide range they do not work with the same precision as the classical applanation tonometer.
\end{abstract}

Traditional applanation tonometry is based on the law of Imbert ${ }^{6}$ and Fick. ${ }^{2}$ According to Goldmann $^{4,5}$ the pressure which is necessary to flatten the cornea gives the intraocular pressure only if the applanated area has a diameter of 2.5 to $4 \mathrm{~mm}$ because it is only in this range that the adhesive force of tears and the force necessary to overcome the rigidity of the cornea are in balance. In recent times, microprocessor controlled tonometers have been developed which operate according to applanation principles but do not fulfil Goldmann's criteria. The Tono-Pen is a little tonometer conveniently held in the hand and operating according to the principle of Mackay and Marg. ${ }^{7}$ It uses only the middle part of the applanation area for pressure measurement, and thus neither the adhesive force of the tears nor the rigidity of the cornea may influence the result. This does not mean however that the intraocular pressure cannot be transferred through the applanated cornea. The airstream of the non-contact tonometers ${ }^{3}$ applanates the cornea without being influenced by the adhesives force of the tears and therefore the requirement of a balance between this adhesive force and the rigidity of the cornea can be disregarded.

An estimation of the practical use of new tonometers should include not only a comparison with traditional tonometry but also a presentation of their physical characteristics.

\section{Materials and Methods}

The new microprocessor controlled tonometer (the non-contact tonometers Pulsair (Fig. 1) and CT-10 (Fig. 2) and the Tono-Pen operating to the principle of Mackay-Marg (Fig. 3) were compared with the hand-held applanation tonometer HAT (Fig. 4) which 


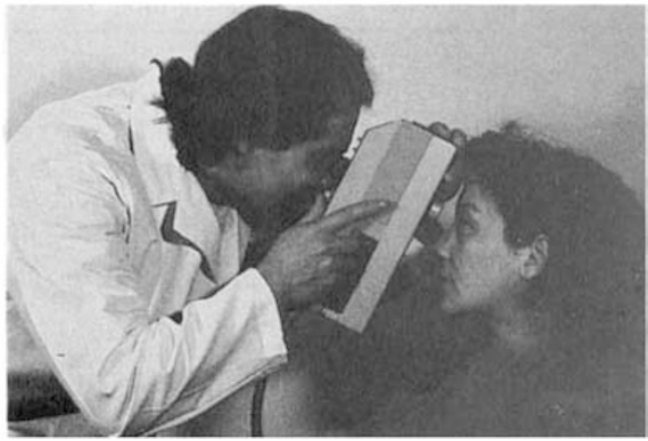

Fig. 1. Pulsair-Tonometer.

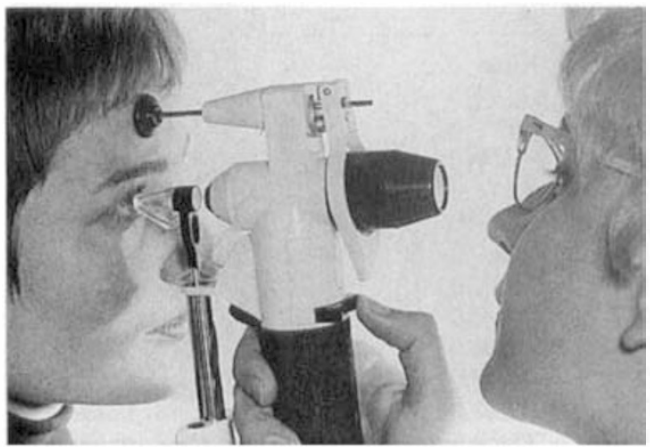

Fig. 3. Tono-Pen.

operates according to the principle of Goldmann. In a randomised sequence two physicians took six readings of every eye of the 99 patients using the different tonometers (see protocol in Fig. 5). The interval between two readings was always three minutes, local anaesthesia was applied just before the first measurement with a contact tonometer. The readings were evaluated by means of the regression analysis in two ways. To determine the accuracy of the instrument regressions and correlations were made between certain microprocessor controlled tonometers on test and the HAT. To determine the precision of each insturment, values were obtained with the tonometer under test using multiple readings on the same eye at different times.

To eliminate the influence of pulse and respiration on intraocular pressure of all three microprocessor controlled tonometers the mean value of three (Pulsair), four (CT-10) or several (Tono-Pen) single measurements were taken as the definite pressure readings.

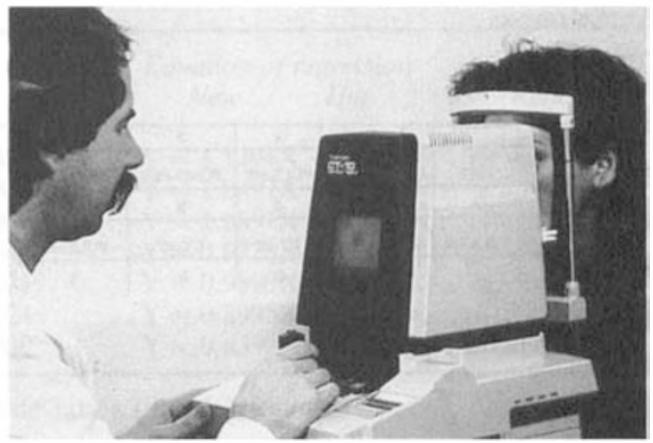

Fig. 2. $C T-10$.

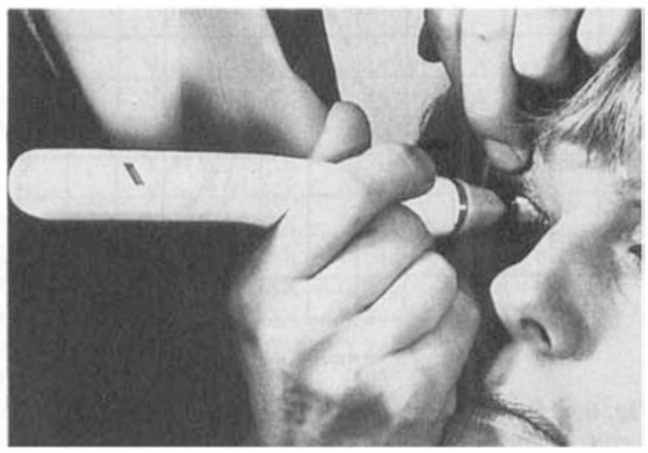

Fig. 4. Hand-held applanation tonometer (HAT).

In Tono-Pen the single values were not used but rather the relative variation coefficient. Only such measurements with variation coefficient of $5 \%$ or less were used. With noncontact tonometers the observer writes down the single values (Pulsair) or the single values are printed on a small label (CT-10).

The airstream of the non-contact tonometers was studied by use of a Constant Temperature Anaemometer. The measuring tip of the anaemometer was moved through the emitted air in horizontal and vertical directions taking steps of $0.25 \mathrm{~mm}$ and keeping the instrument at the normal working distance at the nozzle (Pulsair $14.5 \mathrm{~mm}$ and CT-10 11 $\mathrm{mm})$. Because the profile of airstream alone cannot lead to precise information about the corneal figuration at the moment when pressure value is taken, we therefore installed a camera over a special interface with the CT-10 and a flash light generator in order to take photographs of the corneal surface during the deforming process. 
Atypical protocol

Patient: Any Person

\begin{tabular}{l|c|c|c|c|c|c} 
Measurement No. & 1 & 2 & 3 & 4 & 5 & 6 \\
\hline Right eye & HAT & CT-10 & HAT & PULSAIR & TONO-PEN & TONO-PEN \\
\hline Physician & A & B & A & A & B & A \\
\hline Left eye & PULSAIR & HAT & HAT & CT-10 & CT-10 & PULSAIR \\
\hline Physician & A & A & A & B & B & A
\end{tabular}

Fig. 5. A protocol label.

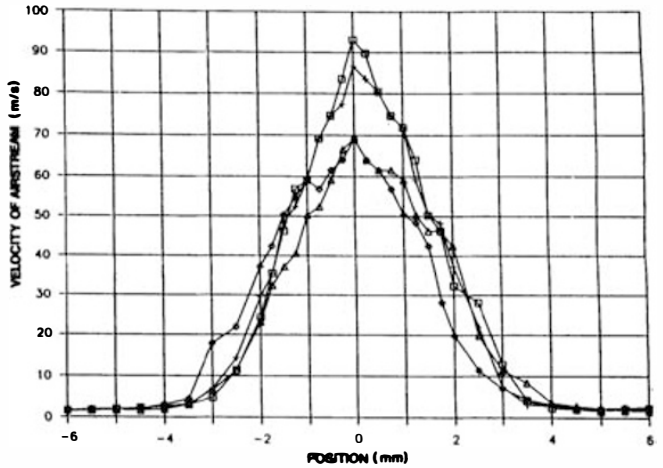

Fig. 6. Profiles of velocity of airstreams measured by a constant temperature anemometer. The anemometer wire was moved horizontally $(\square C T-10, \diamond$ Pulsair) and vertically $(+C T-10, \triangle$ Pulsair) to the emitting direction of the airstream.

\section{Results}

The airstream study

The non-contact tonometers produced a conically formed airstream (Fig. 6) with top speeds of $93 \mathrm{~m} / \mathrm{s}$ (CT-10) and of $69 \mathrm{~m} / \mathrm{s}$ (Pulsair). At the moment of pressure measurement the airstream applanates the cornea exactly. The diameter of the applanated circle is $4 \mathrm{~mm}$ (Fig. 7).

\section{The clinical study}

In a sequence of four Pulsair or three CT-10 measurements the single values should either be arranged at random or they should decrease sequentially because of the decreasing effect of repeating measurements on intraocular pressure. With the Pulsair however the contrary is the case. The simple sign test of Dixon and Moon shows that before the cornea is anaesthetised the first value of a sequence is smaller than the mean value of this sequence $(\mathrm{p}<0.01)$. After anaesthesia the

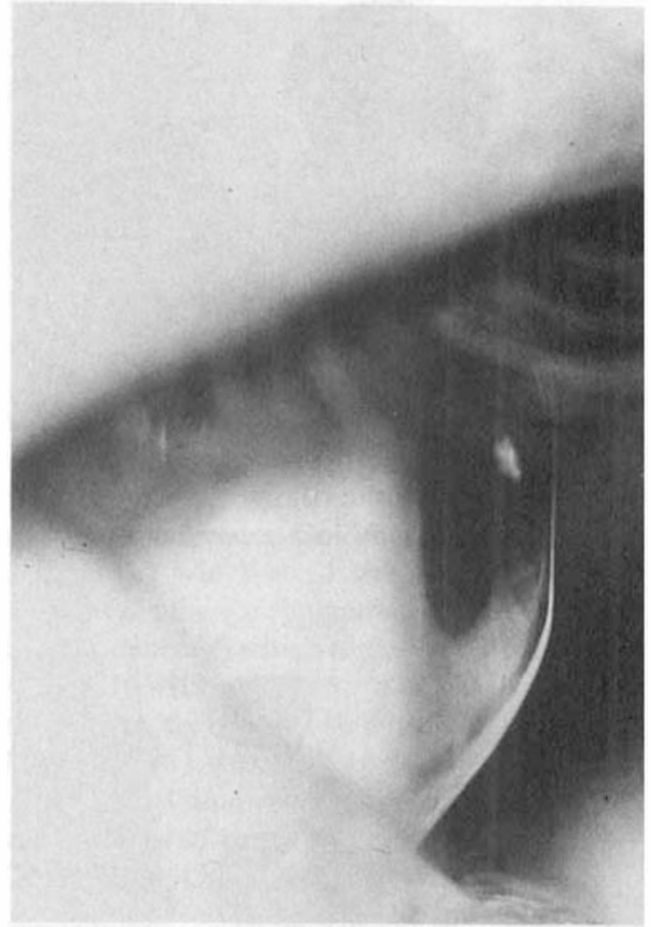

Fig. 7. The applanated cornea at time of pressure measurement. The flashlight was triggered by the microprocessor of the CT-10. The diameter of the applanated area amounts to $4 \mathrm{~mm}$.

first value is distributed randomly as is also the case in CT-10.

The regressions between new tonometers and HAT are shown in Table I and the standard deviations are considerable. There is a statistically significant difference between CT-10 and Pulsair $(\mathrm{p}<0.001)$ and with both tonometers anaesthesia increases the standard deviation (CT-10 $\mathrm{p}<0.001$ and Pulsair $\mathrm{p}<0.005)$. The Tono-Pen especially shows a very high intersection and a very small regression coefficient.

The most precise readings are obtained by the HAT and the worst by Tono-Pen (Table II). This depends on the presence or absence of anaesthesia. While Pulsair gives a bigger deviation before than after anaesthesia, CT-10 shows the opposite effect (Table II). With HAT, CT-10 and Pulsair the standard deviation is significantly smaller if the values are obtained from one observer rather than those obtained by both observers, even 
Table I Correlation between a new tonometer and HAT

\begin{tabular}{|c|c|c|c|c|c|}
\hline Tonometer & $n$ & $r$ & $s$ & $\begin{array}{c}\text { Equation of regression } \\
\text { New }\end{array}$ & Remarks \\
\hline 1 CT-10 & 136 & 0.8606 & 4.5477 & $Y=1.1494 X-2.6187$ & all values \\
\hline 2 CT-10 & 33 & 0.9411 & 3.1840 & $Y=1.0497 X-0.4314$ & before an. \\
\hline 3 CT-10 & 88 & 0.8110 & 5.4033 & $Y=1.0891 X-2.2240$ & after an. \\
\hline 4 Pulsair & 150 & 0.8968 & 3.2641 & $Y=0.9223 X+0.0347$ & all values \\
\hline 5 Pulsair & 49 & 0.9749 & 2.4021 & $Y=0.9899 X-0.6532$ & before an. \\
\hline 6 Pulsair & 93 & 0.8651 & 3.5324 & $Y=0.8995 X-0.1005$ & after an. \\
\hline 7 Tono-Pen & 143 & 0.8414 & 3.7830 & $Y=0.8393 X+5.0657$ & all values \\
\hline
\end{tabular}

$\mathrm{n}$ : number of eyes, $\mathrm{r}$ : correlation coefficient, $\mathrm{s}$ : standard deviation $(\mathrm{mmHg})$, an: anaesthesia.

Table II Autocorrelation of values obtained from one eye with a particular tonometer

\begin{tabular}{|c|c|c|c|c|c|}
\hline Tonometer & $n$ & $r$ & $s$ & Equation of regression & Remarks \\
\hline 1 HAT & 65 & 0.9849 & 1.6617 & $Y=0.9576 X+0.4474$ & A against $B$ \\
\hline 2 HAT & 38 & 0.9925 & 0.9334 & $Y=0.9406 X+0.563$ & A \\
\hline 3 HAT & 37 & 0.9939 & 0.9799 & $Y=1.0090 X-0.5874$ & B \\
\hline 4 CT-10 & 64 & 0.9080 & 2.9179 & $\mathrm{Y}=0.7775 \mathrm{X}+2.3128$ & A against $B$ \\
\hline 5 CT-10 & 42 & 0.9782 & 1.8905 & $Y=0.9598 X-0.1618$ & A \\
\hline $6 \mathrm{CT}-10$ & 35 & 0.8497 & 2.3372 & $Y=1.0163 X-0.8302$ & B \\
\hline 7 CT-10 & 20 & 0.9366 & 1.2209 & $Y=0.9695 X-0.6133$ & before an. \\
\hline $8 \mathrm{CT}-10$ & 65 & 0.9738 & 1.8770 & $Y=0.9638 X+0.2527$ & after an. \\
\hline 9 Pulsair & 78 & 0.9062 & 2.9434 & $Y=1.0292 X-0.4796$ & A against $B$ \\
\hline 10 Pulsair & 44 & 0.9159 & 2.0992 & $Y=0.7240 X+3.2308$ & A \\
\hline 11 Pulsair & 41 & 0.8919 & 2.2330 & $Y=0.8450 X+1.8354$ & B \\
\hline 12 Pulsair & 24 & 0.9331 & 3.6030 & $Y=0.9866 X-0.1530$ & before an. \\
\hline 13 Pulsair & 75 & 0.8586 & 2.5843 & $Y=0.8748 X+1.4240$ & after an. \\
\hline 14 Tono-Pen & 57 & 0.8004 & 3.4969 & $Y=0.9261 X+0.8813$ & A against $B$ \\
\hline 15 Tono-Pen & 45 & 0.7830 & 3.3947 & $\mathrm{Y}=0.6797 \mathrm{X}+6.0182$ & A \\
\hline 16 Tono-Pen & 31 & 0.8285 & 2.7235 & $Y=0.8285 X+1.8751$ & B \\
\hline
\end{tabular}

$\mathrm{n}$ : number of eyes, r: correlation coefficient, s: standard deviation ( $\mathrm{mmHg})$, A and B: Observers, an: anaesthesia.

though there is no difference between the observers. However, the correlation of HAT values obtained by one observer with that obtained by the other shows a smaller deviation than the autocorrelation obtained by the microprocessor controlled tonometers.

\section{Discussion}

The readings of the new microprocessor controlled tonometers correlate with those of conventional tonometers. The applanation tonometry using the Goldmann principle has a greater reliability of values than the new tonometers.

Although CT-10 and Pulsair work by the same principle there are striking differences between both instruments in regard to the sensitivity to temporary alterations of the cornea. The Pulsair especially is very sensitive to such variations.
For control of glaucoma close monitoring of intraocular pressure is considered to be important. This is obviously best achieved if tonometry can be performed independently of an observing physician. The new microprocessor controlled tonometers are a step in this direction. Further research is being undertaken to combine the Goldmann principle with microelectric technology. References
${ }^{1}$ Draeger J, Jessen K: Erfahrungen mit neuen Tonom-
etern und Überlegungen zur Standardisierung.
In: Leydhecker: Glaukomsymposium, $1976 \mathrm{pp}$
183-204, Enke, Stuttgart.
${ }^{2}$ Fick A: (1888) Über Messungen des Druckes im
Auge. Pflügers Arch Ges Physiol 1888, 42: 86.

${ }^{3}$ Grolman B: A new tonometer system. Am J Optom 1972, 49: 646 . 
${ }^{4}$ Goldmann $\mathrm{H}$ and Schmidt T: Über Applanationstonometrie Ophthalmologica 1957, 134: 221-42.

${ }^{5}$ Goldmann $\mathrm{H}$ and Schmidt T: Weitere Beiträge zur Applanationstonometrie. Ophthalmologica 1961, 141: 441-56.
${ }^{6}$ Imbert A: Theorie des ophthalmo-tonometres. Arch Ophthalmol (Paris) 1885, 5: 358-63.

${ }^{7}$ Mackay RS and Marg E: Fast, automatic, electronic tonometers based on an exact theory. Acta Ophthalmol 1959, 37: 495-507. 